\title{
Free-Flight Aerodynamic Testing of a 7 Degree Half-Angle Cone
}

\author{
Andrew Hyslop * , Luke J. Doherty ${ }^{\dagger}$ and Matthew McGilvray ${ }^{\ddagger}$ \\ Oxford Thermofluids Institute, University of Oxford, Oxford, United Kingdom, OX2 OES
}

Free-flight experiments have been conducted in the University of Oxford High Density tunnel, a heated Ludwieg tube, with a 7 degree half angle cone. The goal of the investigation was to examine the potential for the free-flight experimental method to measure purely static aerodynamic coefficients. The tests were conducted at Mach 6 and 7 conditions representative of a hypersonic vehicle's trajectory at $35 \mathrm{~km}$ altitude. The model was designed so that the centre of gravity of the model could be fine-tuned to be at the same position as the centre of pressure, hence minimising the static margin of the model. This resulted in a model that exhibited minimal pitch during a test, thus providing high-quality static aerodynamic data for an unconstrained model. Non-intrusive techniques such as image tracking and on-board inertial measurement units were used to determine accelerations to ultimately determine the aerodynamic forces acting on the cone. Results for lift, drag and pitching moment coefficients were obtained over a range of angles of attack and compared with predictions from a hypersonic panel method code. The experimental and numerical data sets agreed well over the range of angles of attack with the experimental uncertainties remaining below $2.55 \%$ for all coefficients.

\section{Nomenclature}

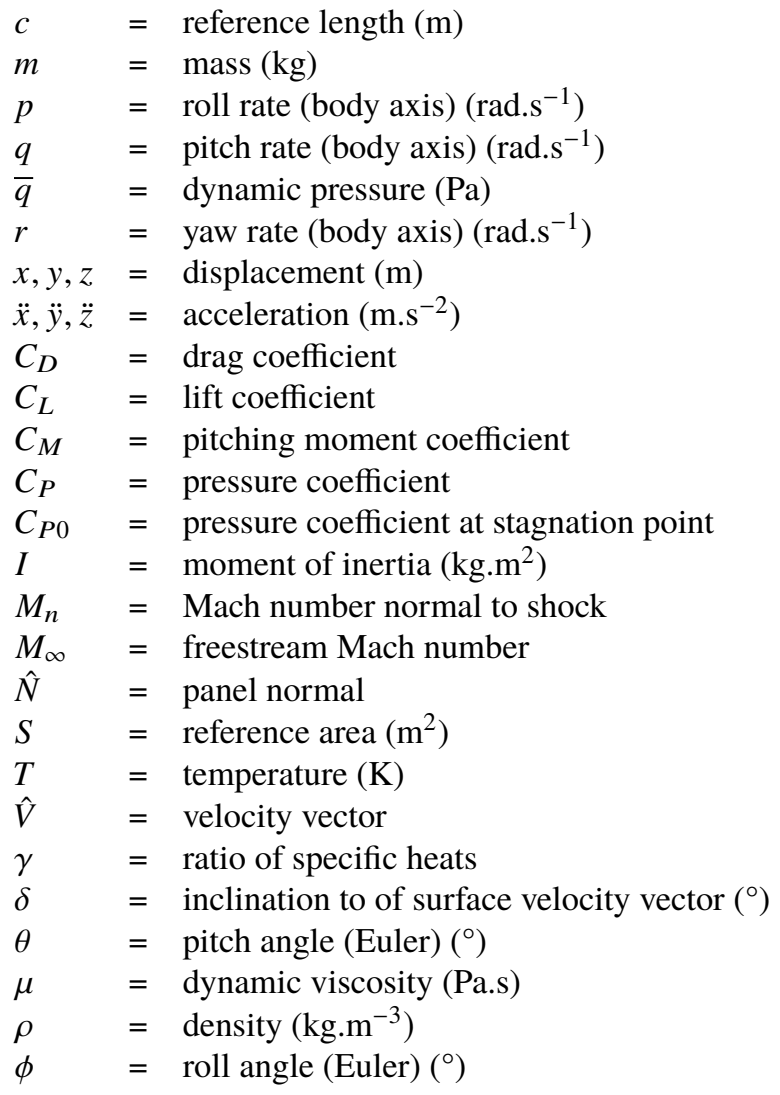

\footnotetext{
*D.Phil. Candidate, Department of Engineering Science

† Senior Research Associate, Department of Engineering Science

$\ddagger$ Associate Professor, Department of Engineering Science
} 


$\begin{array}{lll}\psi & = & \text { yaw angle }(\text { Euler })\left(^{\circ}\right) \\ \text { Subscripts } & \\ f \quad= & \text { fluid } \\ m & = & \text { model } \\ v & = & \text { vehicle }\end{array}$

\section{Introduction}

$\mathrm{T}$ HE knowledge of a vehicle's aerodynamic force coefficients over its flight map is essential for evaluating its performance. The definition of an aircraft's performance often varies depending on its classification, for example; it is highly desirable that a hypersonic boost-glide vehicle has a high lift-to-drag ratio over the extent of its trajectory to maximise its range. Therefore in the design phase, pre-requisite predictions of an aircraft's force coefficients are required before going ahead with costly flight testing. Often these force coefficients are initially predicted using numerical simulations, however, experimental validation is still required to gain confidence in the simulations. In general, the creation of a high-fidelity, nose-to-tail numerical simulation of hypersonic vehicles is difficult and computationally expensive [1] [2]. Validation of such simulations requires high-quality experimental data, however, the measurement of forces in short duration hypersonic facilities at flight representative conditions still poses many challenges to researchers.

The most common method of determining aerodynamic forces in wind tunnel facilities is to mount a model on a semi-rigid sting and measure forces and moments using a force balance. Complexities can occur with multi-component balances due to the often coupled nature of the force signals across the different axes. Furthermore, in short duration hypersonic facilities with test of times in the order of milliseconds to tens of milliseconds, stress waves within the model and supporting structure do not reach equilibrium making it difficult to measure quasi-static aerodynamic forces. Therefore, it is important that the dynamic response of the sting-model system is shorter than the available test time which is often difficult to achieve in hypersonic facilities [3]. Mee et al. [4] developed a rigid 3 component force balance for measurements in shock tunnels using the stress wave technique but accurate forces were still difficult to obtain, even when using advanced deconvolution techniques. The presence of the sting also results in undesirable drag and also produces unrealistic flow fields downstream of the model. Pick [5] showed that the interference effects of a sting is very influential at angles of attack of 15 degrees or greater for sharp cones in hypersonic flow.

Removing the sting and allowing the model to move unconstrained in the flow in six degrees of freedom is known as the free-flight technique. A typical methodology of a free-flight test in short duration facilities is as follows: the model is released prior to arrival of the test flow, the flow is initiated over the model which allows it to move as it would in flight. As the model is not constrained, non-intrusive methods of measuring accelerations, rather than forces, are required. Using this technique removes the complex structural dynamic considerations that must be taken into account for sting based experiments as well as the undesirable sting-flow-field effects. Free-flight has been successfully demonstrated by Kennell [6, 7] with ESA's HEXAFLY INT EFTV geometry and Hyslop [8] for Reaction Engines' Skylon spaceplane. For these experiments, low inertia models were used which allowed the model to freely pitch over a wide range of angles of attack during a test and hence providing large sweeps of aerodynamic coefficient data in a single run. With the large amounts of pitching during the test, the aerodynamic coefficients will include some dynamic influence in the measurement. The current work uses high inertia models with minimal static margin to reduce the magnitude of pitching seen during a test. This results in a purely static free-flight test analogous to a static force balance experiment without the sting.

This paper presents the methodology for obtaining static aerodynamic force coefficients using the free-flight experimental technique in the University of Oxford High Density Tunnel (HDT) applied to a 7 degree half-angle cone model. Data was acquired both using high speed video and an on-board IMU (Inertial Measurement Unit). Novel to this work is the ability to fine tune and measure (to $0.1 \mathrm{~mm}$ accuracy) the model's centre of gravity to minimise the static margin so that the model stays at constant angle of attack for the duration of the test time. Furthermore, by using a simple 7 degree cone, this work has lead to the validation of the free-flight technique for the determination of static aerodynamic coefficients against standard analytical models. This gives confidence in the technique for testing more complex geometries in the future. 


\section{Static Free-Flight Scaling}

In order to obtain flight realistic aerodynamic forces for wind tunnel experiments, it is necessary to scale the free-stream flow properties to obtain similitude. If the experimenter is also interested in measuring the dynamic aerodynamic coefficients, the model's inertial properties will also need to be scaled. This section discusses the scaling laws for conducting static free-flight tests as applied to model properties and freestream test conditions.

\section{A. Freestream Scaling}

For aerodynamic free-flight testing, there are two options available to the researcher for scaling the free-stream conditions. For low-speed incompressible flow, Froude scaling is typically used. Scaling based on the Froude number (defined as the ratio of inertial to buoyancy forces), preserves the gravitational forces acting on the model, thereby allowing the kinematics between flight and tunnel testing to be matched such as angle of attack similitude. In the hypersonic regime, compressibility effects dominate and hence it becomes vital that Mach number is matched between tunnel experiments and flight. Mach scaling also requires that the free-stream Reynolds number is matched between flight and tunnel so that features such as viscous effects and boundary layer transition are preserved. As this work is entirely within the hypersonic flow regime, Mach scaling was used to obtain the suitable free-stream conditions for testing.

If both Reynolds and Mach number are matched, the following equation can be derived by taking ratios of Reynolds number (where subscript $\mathrm{m}$ denotes the model, subscript $\mathrm{v}$ the full-scale vehicle and $\rho_{f}$ is the fluid density):

$$
\left(\frac{\rho_{f} T^{\frac{1}{2}}}{\mu}\right)_{m}=\left(\frac{\rho_{f} T^{\frac{1}{2}}}{\mu}\right)_{v}
$$

With knowledge of the model scale and flight trajectory point of interest, all flow properties on the right hand side of Eq. 1 1 are fixed. Therefore, the left hand side describes a test envelope that the test facility must achieve in order to achieve similitude with Mach scaling. This scaling technique assumes that high temperature effects such as flow chemistry play a secondary role in aerodynamic experiments and as a consequence, stagnation enthalpies are not replicated in Mach scaling. For more information on scaling for free-flight experiments, see Wolowicz [9].

For the series of experiments in this work, two trajectories were used which are representative of vehicles in the hypersonic regime [10]. The two conditions were at Mach 6 and 7, both at a representative altitude of $35 \mathrm{~km}$. Section III B discusses the scaling applied to these trajectory points and Tables 1 and 2 present both the in flight and scaled free-stream conditions.

\section{B. Model Scaling}

For these series of experiments, the primary concern was for the measurement of static aerodynamic coefficients and hence the model's mass and inertia were optimised to allow for this. In typical aerodynamic force measurement experiments, the model is rigidly mounted using a sting so that it is fixed at a single angle of attack for the entire test. A comparable test in free-flight is one in which the model exhibits minimal pitching and remains at a constant angle of attack. There are two approaches which could be taken to achieve this. The first is to design a model that has very large inertia so that during a test, the forces acting on the model result in very small angular (and linear) accelerations. The main difficulty with this approach is that as the accelerations are small, they are difficult to measure and uncertainties in the resulting force coefficients are large.

The second approach and the one used in this work is to consider the nature of the aerodynamic forces acting on a free body in free-flight. As the fluid passes over the body during a test, pressure and shear components are exerted on the surface of the body. Integrating the shear and pressure components over the body results in the overall aerodynamic force acting on the body. This integrated force can be considered to be a single force vector at the average integrated location, often referred to as the centre of pressure $(\mathrm{CoP})$. The force vector is usually split into two orthogonal forces referred to as lift and drag. Finally, as the body is free to move in space, its weight needs to be considered which acts as a single vector through the centre of gravity $(\mathrm{CoG})$. The position of the $\mathrm{CoG}$ relative to the CoP determines the stability of the body in flight. If the $\mathrm{CoG}$ is fore of the CoP, the body is statically stable and will tend to pitch towards an equilibrium position if perturbed. If the $\mathrm{CoG}$ is aft of the $\mathrm{CoP}$, the body is statically unstable and will pitch away from an equilibrium position which is often undesirable as it makes the body difficult to control. In both of these cases, the body will exhibit increasing pitch rates as the distance between the $\mathrm{CoP}$ and $\mathrm{CoG}$ is increased. If a model is designed so that the $\mathrm{CoP}$ is at the same position of the $\mathrm{CoG}$, there will be no aerodynamic pitching moment and hence could be 
considered as a static free-flight test.

In practice, this requires: (1) a method of predicting the CoP location, (2) a model design that allows for fine tuning of the CoG location and (3) a setup that can accurately measure the CoG location before a test. It should be noted that in this methodology of scaling that the model design is independent of mass and moment of inertia and so the experimenter is not limited by material choice as they would be if scaling for dynamic experiments.

\section{Experimental Setup}

\section{A. Facility and Test Infrastructure}

The experiments were conducted in the University of Oxford High Density Tunnel located within the Oxford Thermofluids Institute. The facility was operated as a heated Ludwieg tube with the Mach 6 and 7 contoured nozzles of exit diameter $350 \mathrm{~mm}$, producing a core flow of $280 \mathrm{~mm}$ at nozzle exit. The facility is typically operated at total pressures up to 50 bar but is rated for fill pressures of 275 bar. For further details on the HDT operation and measurement of freestream conditions, see McGilvray et al. [11] and Wylie et al. [12]. This facility was selected for these experiments because it offers sufficient steady flow duration $(\sim 50 \mathrm{~ms})$ to allow for the measurement of quasi-static forces in free-flight at flight representative conditions.

Shown in Fig. 1. a catcher net and model drop mechanism were designed for the HDT. The catcher mechanism consists of two aluminium rings which are interwoven through 32 uniformly spaced holes with $1.5 \mathrm{~mm}$ diameter Dyneema cord to form a net. The upstream ring is held at a $30^{\circ}$ inclination which is designed to deflect the model towards the tunnel floor upon contact which is lined with multi-layer foam. The purpose of the catcher rings is two-fold, firstly to reduce the amount of damage taken by the model by providing a soft impact surface, and secondly to prevent the model entering the facility dump tank where it would be difficult to retrieve.

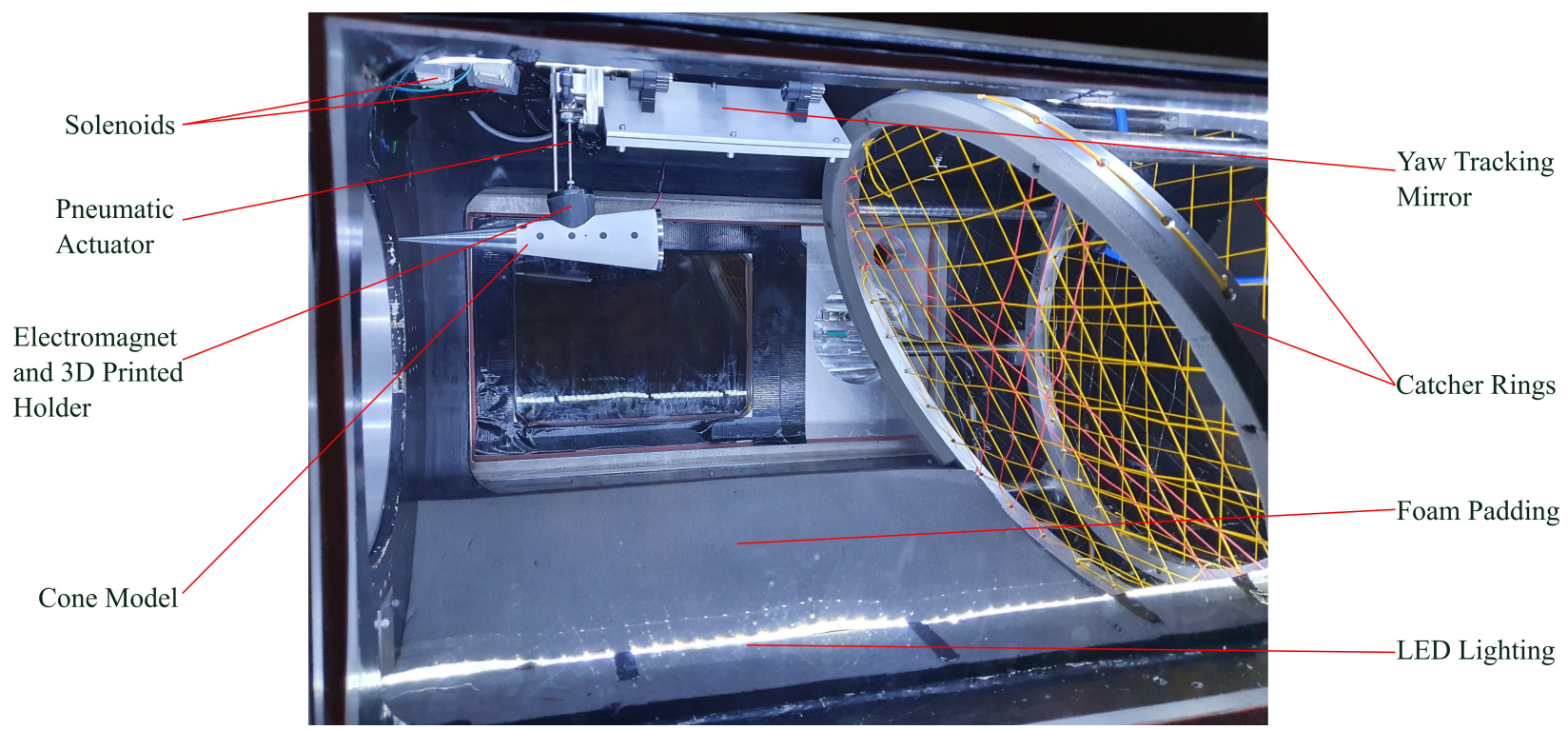

Fig. 1 Free-flight drop mechanism and catcher rings in the HDT test section.

The drop mechanism was designed to release the model into the core flow prior to the arrival of test flow. The system consisted of a $25 \mathrm{~mm}$ electromagnet connected to a $50 \mathrm{~mm}$ stroke length SMC pneumatic actuator which removes the electromagnet from the core flow once the model is released. The actuator and electromagnet were controlled by a bespoke electrical control unit which upon receiving a TTL pulse from the tunnel, triggers the electromagnet to release the model and powers two solenoids to retract the actuator, removing the magnet from the oncoming flow. The entire drop mechanism is mounted on a linear rail so that the axial position of the drop can be changed if required. A 3D printed holder was used to house the electromagnet and was shaped to be conformal to the cone model, allowing for repeatable alignment in roll and axial position of the model prior to the test. Different holders were printed to allow for the initial angle of attack to be set in two degree increments. A steel rod is inserted into a tightly tolerance hole in the 
holder which fixes the initial position in yaw. The printed holder is shaped to position the electromagnet directly above the centre of gravity of the model to minimise any exerted rotational inertia on the model when it is released and hence provided a consistent and repeatable model release.

Attached to the top plates of the drop mechanism through angled bracket mounts is a mirror which allows for optical tracking on the yaw axis of the model. To improve optical visibility of the model during the test, four 400 lumen LED strips were attached internal to the test section.

\section{B. Test Conditions}

Scaling a high speed vehicle for a free-flight experiment in a wind tunnel typically requires compromise on matching all the non-dimensional parameters of interest. Within the hypersonic regime, matching the Mach number and therefore the effects of compressibility is of primary importance. As a consequence, this results in a mis-match in Froude number which is important for kinematic similarity. As a starting point for scaling, a suitable model length needs to be chosen to give the geometric scaling factor. For this work, the model scale was dictated by the requirement that the entire model remain within the core flow diamond (biconic) of the HDT nozzles throughout the duration of steady test flow. This resulted in a model that was geometrically scaled to 1:16th of the design scale; the total length of the model was 250 $\mathrm{mm}$ (with the assumption that a full scale vehicle is on order of length $4 \mathrm{~m}$ ) [13].

Two test conditions were used replicating a hypersonic vehicle at Mach 6 and 7 at an altitude of $35 \mathrm{~km}$. With knowledge of the trajectory points (Section IIA) and model scale, Eq. 1 can be used to determine the tunnel test conditions. The conditions obtained during a test were surveyed prior to the experiments using a rake instrumented with pressure transducers and thermocouples, providing freestream pitot pressure and temperature traces. The calculated test conditions are provided in Tables 1 and 2.

Table 1 Mach 6 Flight vs scaled tunnel condition.

\begin{tabular}{lcc}
\hline \hline & HDT Test Flow & Flight \\
\hline Altitude $(\mathrm{km})$ & 35 & 35 \\
Mach Number & $6.08 \pm 0.7 \%$ & 6 \\
Velocity $\left(\mathrm{ms}^{-1}\right)$ & $939.1 \pm 3.8 \%$ & 1851.7 \\
Density $\left(\mathrm{kgm}^{-3}\right)$ & $0.0743 \pm 11.1 \%$ & 0.0082 \\
Static Pressure $(\mathrm{Pa})$ & $1299.6 \pm 4.8 \%$ & 558.9 \\
Total Pressure $(\mathrm{kPa})$ & $2051.9 \pm 0.1 \%$ & 882.4 \\
Static Temperature $(\mathrm{K})$ & $61.0 \pm 6.8 \%$ & 237.1 \\
Total Temperature $(\mathrm{K})$ & $500 \pm 8.7 \%$ & 1943 \\
Dynamic Viscosity $(\mu \mathrm{Pas})$ & $4.4 \pm 6.8 \%$ & 15.3 \\
Unit Reynolds Number $\left(10^{6} \mathrm{~m}^{-1}\right)$ & $15.9 \pm 13.5 \%$ & 1.0 \\
Reynolds Number $\left(10^{6}\right)$ & $3.98 \pm 13.5 \%$ & 3.98 \\
\hline \hline
\end{tabular}


Table 2 Mach 7 Flight vs scaled tunnel condition.

\begin{tabular}{lcc}
\hline \hline & HDT Test Flow & Flight \\
\hline Altitude $(\mathrm{km})$ & 35 & 35 \\
Mach Number & $7 \pm 0.8 \%$ & 7 \\
Velocity $\left(\mathrm{ms}^{-1}\right)$ & $954.7 \pm 4.1 \%$ & 2160 \\
Density $\left(\mathrm{kgm}^{-3}\right)$ & $0.0646 \pm 11.3 \%$ & 0.0082 \\
Static Pressure $(\mathrm{Pa})$ & $858.0 \pm 4.8 \%$ & 558.9 \\
Total Pressure $(\mathrm{kPa})$ & $3551.9 \pm 0.1 \%$ & 2313.8 \\
Static Temperature $(\mathrm{K})$ & $46.3 \pm 6.9 \%$ & 241.5 \\
Total Temperature $(\mathrm{K})$ & $500 \pm 8.7 \%$ & 2560.1 \\
Dynamic Viscosity $(\mu \mathrm{Pas})$ & $3.3 \% \pm 6.9$ & 15.2 \\
Unit Reynolds Number $\left(10^{6} \mathrm{~m}^{-1}\right)$ & $18.6 \pm 13.7 \%$ & 1.16 \\
Reynolds Number $\left(10^{6}\right)$ & $4.65 \pm 13.7 \%$ & 4.65 \\
\hline \hline
\end{tabular}

\section{Experimental Model}

The experimental model was geometrically scaled to be representative of a full-scale hypersonic vehicle, resulting in a 1:16th scale model. The model was designed to be modular to allow for easy instrumentation and ballasting. Therefore, it was manufactured so that it could be split into two halves for ease of access. The two halves form a male thread near the front when joined together so that nose tips of different materials (for ballasting purposes) and nose radii $(1.25,2.5$ and $5 \mathrm{~mm})$ could be used. For these sets of experiments, the cone halves and rear were machined from steel and the nose tips were aluminium.

The most important aspect of the experimental model was the ability to change the centre of gravity location. As the model was made of steel, the ballasting was achieved through tungsten disks whose position could be adjusted internally in the model using threaded steel rods. The CoP of the model was predicted prior to testing using Modified-Newtonian Theory to be $83.6 \mathrm{~mm}$ from the base. The tungsten ballast allowed for the CoG to be shifted between $80.1 \mathrm{~mm}$ to 85.7 $\mathrm{mm}$. Table 3 presents the inertial and geometric properties of the cone model. The centre of gravity was measured using a moment balance, whereby the model was suspended using two strings. The tension in the upstream string was measured using a load cell. This setup allowed the CoG to be determined to $+/-0.1 \mathrm{~mm}$. The moment of inertia was not measured directly but determined through the Computer Aided Design software Solidworks. As these were static aerodynamic tests, an accurate measurement of moment of inertia is not required as the model exhibits minimal pitch.

Internal to the model was an on-board Data Acquisition system (DAQ) which contained a six degree-of-freedom inertial measurement unit (IMU) consisting of accelerometers and gyroscopes. The board was mounted on a 3D printed holder which fitted conformal to the internal shape of the cone halves, fixing it rigidly in place.

For the exterior, the model was painted white and a laser-cut stencil was used to airbrush black dots on the model with a tolerance in centre point location of $+/-0.2 \mathrm{~mm}$ for the purpose of image tracking. The black and white paint created a high contrast finish enabling the image tracking algorithm to work more effectively in post-processing. Also painted on the model were two ' $T$ ' shapes to mark the point where the electromagnet attaches to the model. It is important for the drop mechanism that the release point was at the $\mathrm{CoG}$ of the model to prevent pitching during the drop free-fall period. Figure 2 shows the constituent components of the cone model. 


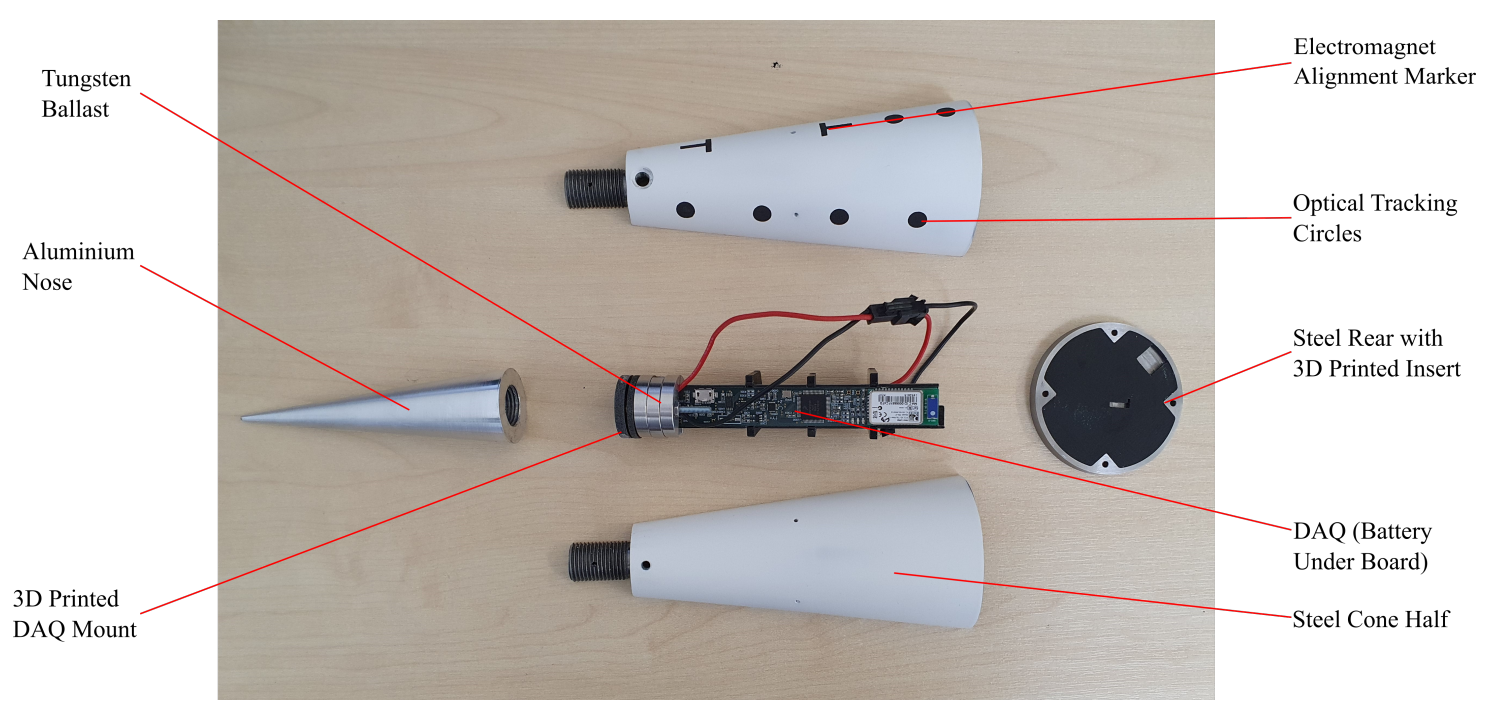

Fig. 2 Constituent components of the 7 degree cone model.

Table 3 Model inertial and geometric properties

\begin{tabular}{lc}
\hline \hline Length $(\mathrm{mm})$ & $250 \pm 0.2$ \\
Cone Half-Angle $\left(^{\circ}\right)$ & $7 \pm 0.03$ \\
Nominal Nose Radius $(\mathrm{mm})$ & $1.25 \pm 0.1$ \\
Base Area $\left(\mathrm{m}^{2}\right)$ & $3.2 \times 10^{-3} \pm 0.01$ \\
Centre of gravity $(\mathrm{mm})$ & $165.7 \pm 0.1$ \\
Mass $(\mathrm{g})$ & $669.73 \pm 0.01$ \\
Moment of Inertia $\left(\mathrm{kgm} \mathrm{m}^{2}\right)$ & $2.15 \times 10^{-3}$ \\
\hline \hline
\end{tabular}

\section{On-board Data Acquisition System}

The on-board inertial measurement instrument (hereby referred to as the DAQ) were designed and built by UNSW Canberra. They consisted of an integrated 3-axis accelerometer and 3-axis gyroscope, non-volatile memory, a Bluetooth radio module and a microcontroller. These components were integrated on a single printed circuit board with planform dimensions of $\sim 114 \mathrm{~mm} \times 12 \mathrm{~mm}$. This measurement system has successfully demonstrated the ability to collect accurate acceleration and angular velocity data necessary for hypersonic wind tunnel model aerodynamics [7, 14]. The IMU was a single chip device with an accelerometer full scale range of $+/-16 \mathrm{~g}$ and a data output rate of $4 \mathrm{kHz}$. The full scale range of the gyroscope was $+/-2000 \mathrm{deg} / \mathrm{s}$ with a data output rate of $8 \mathrm{kHz}$. Non-linearity of the accelerometer and gyroscope axis is reported to be $0.1 \%$ and $0.5 \%$ respectively. For this work a sample rate of $8 \mathrm{kHz}$ was used which allowed for $\sim 320$ samples over the $40 \mathrm{~ms}$ duration of the experiment. Triggering of the instrument was achieved by detection of sustained free-fall upon model release.

\section{E. Optical Setup}

For this work, image tracking was conducted on both the yaw and longitudinal axis. The longitudinal axis was imaged by a FASTCAM AX200 high-speed camera set to a frame rate of $6400 \mathrm{fps}$ with 1,024 x 1,024 pixel resolution at a focal length of $50 \mathrm{~mm}$ at $\mathrm{f} 2.8$. The camera faced directly through the test section windows, imaging the longitudinal plane of motion of the model. To fully capture the free-fall of the model, a pre-trigger of 500 frames was used and a total of 2180 frames recorded, activated by a TTL pulse from the facility prior to the test. A FASTCAM UX100 high-speed camera was used to track the yaw axis with a frame rate $1000 \mathrm{fps}$ resulting in a resolution of $1,280 \times 1,024$ pixels. The yaw axis was imaged through a reflection of a mirror on the roof of the test section and was mainly used to confirm the model was exhibiting minimal yaw during a test. Six 1000 lumen torches were used to front light the model, providing adequate spacial uniformity of light. The two cameras were positioned as such that both systems could 
operate concurrently during a test with neither system obstructing the field of view of the cameras. A few experiments were conducted with schlieren imaging to confirm that the model was in the core flow during the course of a test. In order to achieve this, both of the optical tracking cameras were removed. For the schlieren, a conventional Z-type setup with horizontal knife edge was used to visualise the density gradients in flow field around the cone model. The light was emitted continuously from a Luminous PT-120-TE green LED as the camera's detector is particularly sensitive to this wavelength region. The flow was imaged with the FASTCAM AX200 high-speed camera at $6400 \mathrm{fps}$.

\section{Numerical Work}

For this work, a panel method aerodynamics code was created to determine the aerodynamic coefficients of the cone geometry. This builds upon the work of Donaldson [15], expanding on the functionality and creating an aerodynamic tool in the MATLAB language domain. The purpose of the code was twofold; the experimental work validates the aerodynamics code so that it can be used in future for more complex geometries and secondly, the rapid nature of the code means it can be tied to trajectory calculations. The aerodynamics code is written entirely in MATLAB and best simulates trajectories that are in the continuum regime. The code requires an input of the freestream conditions to begin the analysis. This is either calculated by inputting a trajectory point (altitude) and Mach number so that freestream conditions can be calculated through 'US Standard Atmosphere 1976' [16] or utilizing a condition calculator that outputs HDT test conditions. Following this, the attitude (roll, pitch and yaw) of the geometry of interest are inputted.

This section discusses the principles on which the code operates and the methods it uses for deriving key values for calculations.

\section{A. Geometry}

Geometry is processed in the form of a tessellated triangular mesh with the individual faces referred to as 'panels'. For simplicity and speed, meshes are input using the STL format due to the wide availability of meshing preprocessing tools. Figure 3 shows an example of the 7 degree cone mesh used in the processing. The preprocessing closely follows the methods used by Donaldson [15]. Upon importing the geometry into MATLAB, the mesh panels are processed so that the surface area, geometric centre and unit normal are calculated. The user also manually inputs a reference length, overall surface area and location of centre of gravity for later calculations.

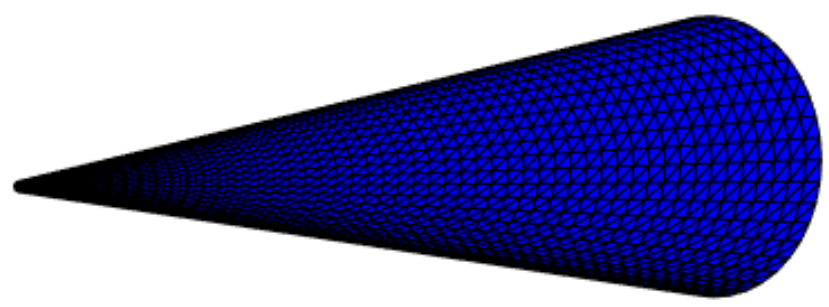

Fig. 3 Triangulated mesh of a 7 degree cone

To determine the surface pressures of each of the panels, the panels are first processed to determine their orientation relative to the freestream velocity vector. This angle, denoted $\delta$, is subsequently used for the calculation of pressure coefficient. The equation for panel orientation to freestream is given by (where $\hat{N}$ is a panel normal and $\hat{V}$ is the velocity vector): 


$$
\delta=\cos ^{-1}\left(\frac{\hat{N} \cdot \hat{V}}{|\hat{N}| .|\hat{V}|}\right)=\cos ^{-1}(\hat{N} \cdot \hat{V})
$$

\section{B. Aerodynamics}

To determine the pressure coefficient on each of the panels, the code uses widely established panel inclination methods which relate the inclination angle $\delta$ and freestream properties to the pressure coefficient. Firstly, using the value of $\delta$, a panel is determined to be either windward (exposed to the flow) or leeward (hidden from the flow). If $|\delta|<90$ the panel is treated as windward, otherwise it is assigned to be leeward. The user has different options to calculate the pressure coefficient. The windward methods are given by:

1) Modified Newtonian Flow - the pressure exerted on a vehicle is due to the loss of momentum of the fluid normal to the vehicle's surface. The modified version includes the stagnation pressure of the freestream as a correction factor.

$$
C_{p}=C_{P 0} \sin ^{2} \delta
$$

2) Tangent Cone - a semi-empirical method based on a three-dimensional flow field determined by constructing an equivalent cone tangent to the centroid of a panel and then calculating the surface pressures behind a conical shock. This code uses an empirical method derived from Cruz [17] and is suited towards slender bodies of revolution:

$$
C_{p}=\frac{48 M_{n}^{2} \sin ^{2} \theta}{23 M_{n}^{2}-5}
$$

The leeward methods are given by:

1) Newtonian - all leeward panels have the same surface pressure as the freestream pressure, resulting in a pressure coefficient of zero.

$$
C_{p}=0
$$

2) High Mach base pressure (full vacuum) - a total vacuum is formed in the wake region of the model.

$$
C_{p}=\frac{-2}{\gamma M^{2}}
$$

3) High Mach base pressure (70\%) - recirculation and expansion in the wake region dive the surface pressures above a full vacuum.

$$
C_{p}=\frac{-1}{M^{2}}
$$

The user can select which methods to use to calculate the surface pressure coefficients for a given model. For blunt bodies, Modified-Newtonian theory is often more suitable than Tangent-Cone due to the nature of the flow and shock patterns around a blunt body. For this work, Tangent-Cone is used with a toggle which switches to Modified-Newtonian for blunt areas such as the blunted nose. $70 \%$ base pressure is selected for the leeward panels due to its good agreement with experimental data.

\section{Viscous Effects}

At hypersonic Mach numbers, the interaction between the outer inviscid flow and viscous boundary layer become significant and cannot be discounted. It has been shown in previous studies that viscous shear stresses can account for more than $25 \%$ on hypersonic vehicles [18]. The method in this study accounts for viscous effects by determining the path of streamlines across the surface of a vehicle. These streamlines can then be used to determine the local Reynolds number of a panel and hence correlations can be used to determine local skin friction coefficients, similar to the method applied by Wuilbercq in HyFlow [19].

In order to generate surface streamlines, the surface velocity field is calculated based on Newtonian impact theory in which particles upon collision to a surface, retain their tangential velocity. Streamlines are tracked from the rear of the vehicle and propagated from panel to panel using a 4th order Runge-Kutta numerical method. A streamline is ultimately 
terminated at a stagnation point which can be determined by setting a bound on the dot product between the surface normal and streamline shear vector; these will be tangential at a stagnation point. After a suitable number of streamlines are detected which give good coverage of the vehicle's surface, the running length of each streamline is calculated. This is then interpolated over the mesh so that each panel has a running length value. Figure 4 presents streamlines as calculated for the cone model at 4 degrees angle of attack.

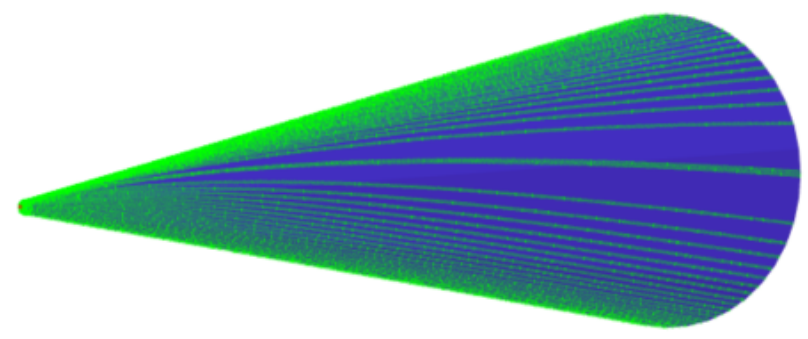

Fig. 4 Calculated streamlines for cone at 4 degrees angle of attack

The streamline running length is ultimately used to calculate the local Reynolds number on each of the panels. However, difficulties arise when calculating the temperature of the flow at the surface of a panel due to the large temperature gradient across the thickness of the hypersonic boundary layer. To correct for this, the code uses the Smart-Meador method [20] to calculate a reference boundary layer temperature. These expressions are then used to calculate the local skin friction coefficients using Eckert's procedure [21] which are derived from experimental skin friction coefficients on a flat plate. It is down to the user to decide whether the flow is laminar or turbulent in the current version of the code. With knowledge of both the inviscid pressure coefficient and skin friction coefficients, the overall aerodynamic coefficients can be calculated.

\section{Data Processing}

Two non-intrusive independent methods of deriving aerodynamic force coefficients were used in this work. Both methods ultimately measure the model's acceleration to determine the instantaneous forces acting on the model during the test time using the model's mass and moment of inertia (Table 3). The forces are then non-dimensionalised by the freestream dynamics pressure (Table 1 and 2 , and a reference area, which in this work was taken to be the cone base area $\left(\mathrm{S}=3.2 \times 10^{-3} \mathrm{~m}^{2}\right.$ ). The reference length used in the calculation of pitching moment was the model length.

$$
C_{L}=\frac{m \ddot{z}}{\bar{q} S}, \quad C_{D}=\frac{m \ddot{x}}{\bar{q} S}, \quad C_{M}=\frac{I_{y y} \ddot{\theta}}{\bar{q} S c}
$$

\section{A. IMU Data Processing}

Before discussing the details of processing IMU accelerations and angular velocities, it is first necessary to define the co-ordinate systems used in the processing. There are two frames of references used in this work; the first is known as the Inertial/Earth coordinate system which is defined as having the $+z$ axis towards the ground, $+x$ axis pointing upstream towards the facility nozzle and the $+y$ axis orthogonal to both to form a right-hand coordinate system. This frame of reference is what all global force coefficients are defined relative to.

The second co-ordinate system is referred to as the body frame of reference. This is defined relative to the model as $+x$ towards the nose of the vehicle, $+z$ towards the bottom of the vehicle with $+y$ completing the orthogonal system. The IMU located on the model measures accelerations and angular velocities in the body frame of reference and hence during a test, this frame of reference is moving relative to the Inertial frame. To process the raw IMU data, the data is filtered with a $6^{\text {th }}$ order low pass Butterworth filter with a cut off frequency of $500 \mathrm{~Hz}$ to remove high frequency noise. The body angular rates are then transformed to Euler angular rates, using Eq. 9, allowing the Euler angles to be determined via numerical integration of Euler-rates. Using the Euler angles, the linear accelerations measured by the 
IMU are transformed to the inertial frame of reference according to Eq. 10 . Finally the aerodynamic coefficients can be obtained using Eq. 8 .

The rotation matrix for the angular rates and accelerations are as follows (where $\mathrm{c}, \mathrm{s}$ and $\mathrm{t}$ are cosine, sine and tangent functions respectively):

$$
\begin{gathered}
{\left[\begin{array}{c}
\dot{\phi} \\
\dot{\theta} \\
\dot{\psi}
\end{array}\right]=\left[\begin{array}{ccc}
1 & s(\phi) t(\theta) & c(\phi) t(\theta) \\
0 & c(\phi) & -s(\phi) \\
0 & \frac{s(\phi)}{c(\theta)} & \frac{c(\phi)}{c(\theta)}
\end{array}\right]\left[\begin{array}{l}
p \\
q \\
r
\end{array}\right]} \\
{\left[\begin{array}{c}
\ddot{X} \\
\ddot{Y} \\
\ddot{Z}
\end{array}\right]_{E}=\left[\begin{array}{ccc}
c(\psi) c(\theta) & c(\psi) s(\phi) s(\theta)-c(\phi) s(\psi) & s(\phi) s(\psi)+c(\phi) c(\psi) s(\theta) \\
c(\theta) s(\psi) & c(\phi) c(\psi)+s(\phi) s(\psi) s(\theta) & c(\phi) s(\psi) s(\theta)-c(\psi) s(\phi) \\
-s(\theta) & c(\theta) s(\phi) & c(\phi) c(\theta)
\end{array}\right]\left[\begin{array}{c}
\ddot{X} \\
\ddot{Y} \\
\ddot{z}
\end{array}\right]_{B}}
\end{gathered}
$$
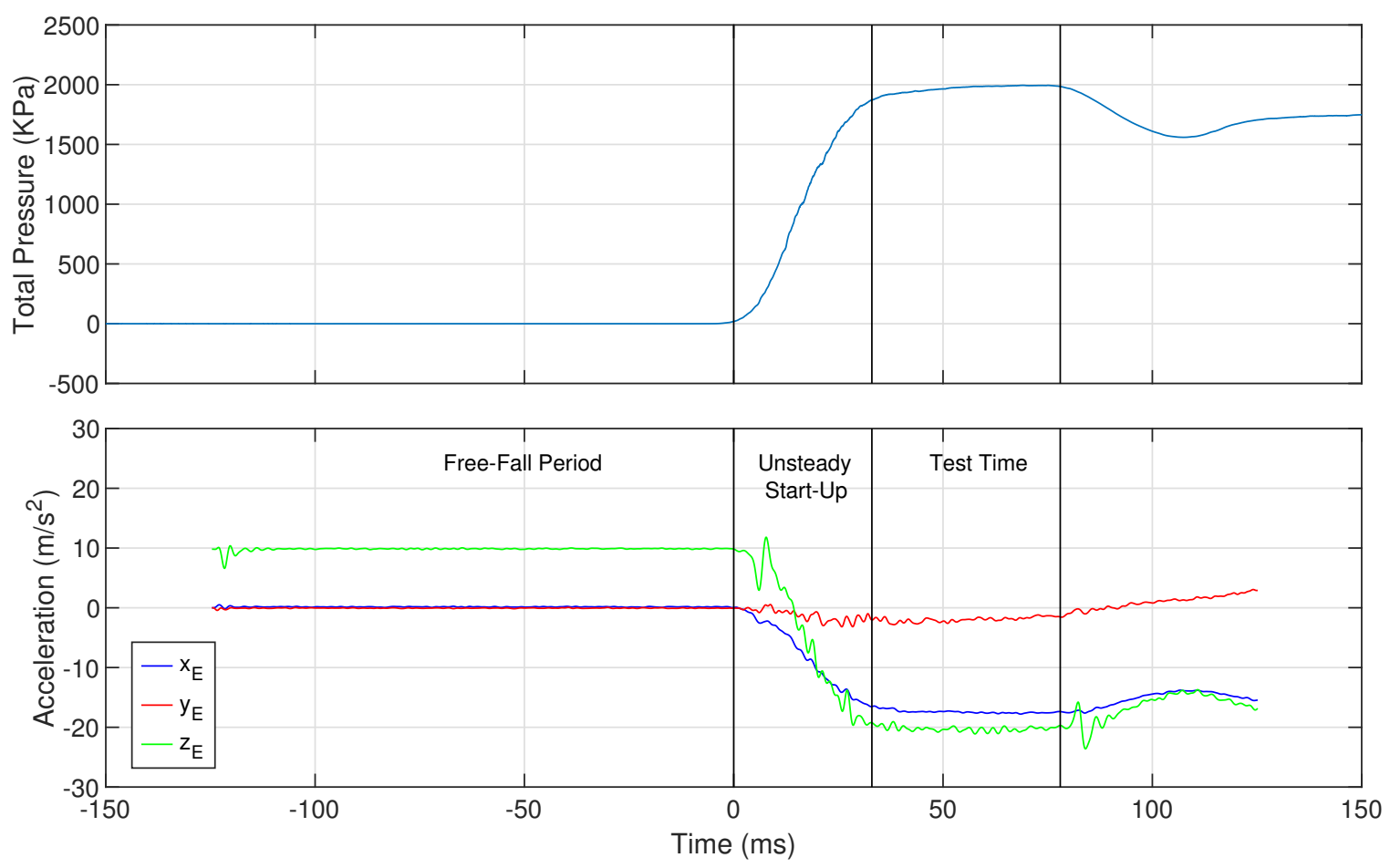

Fig. 5 Example filtered accelerometer accelerations in the Earth frame of reference with HDT total pressure.

Figure 5 presents a plot of filtered IMU accelerations with freestream total pressure for a typical free-flight test. As seen in the figure, the model free-falls for approximately $120 \mathrm{~ms}$ before the onset of flow. During this time the model falls into the centre of the core flow of the tunnel. The tunnel takes $30 \mathrm{~ms}$ to start up before the steady plateau in free stream conditions are reached. It is during this steady plateau that the test time is taken. The filtered accelerations in the direction of lift and drag mimic the plateau in free stream stagnation pressure. The accelerations stay constant during the steady test time due to the minimal pitching exhibited by the model during a test.

\section{B. Image Processing}

Optical tracking was used to measure the model's displacement about the centre of gravity of the model. The method used in this work uses a methodology which finds the centre point of high contrast circles painted on the surface of the model. As seen in Figure 1, for longitudinal tracking (about pitch axis), four black circles are painted on the side of the model which are at known locations relative to the model. Only two circles are required for the algorithm but the extra two circles are for redundancy in the case of damage or non-uniform lighting. The methodology for image tracking is the same as in the work of Hyslop [8] but the steps are briefly summarised as follows: 
1) Apply Gaussian filter to image and subtract from original (High pass filter).

2) Apply Canny filter to image so that only pixels detected as an edge are shown [22].

3) Apply Hough transform to find circles in the image after narrowing the search radius [23].

4) Detect pixels in the proximity of the circle located by the Hough transform.

5) Use subpixel detection on the original image, at the location pixels were detected. Sub-pixel methodology set out in von Gioi [24].

6) Fit a circle to the pixels using linear regression as set out in Laurence et al. [25] and use this equation to find the centre point of the circle.

Following the detection of the centre point of two circles, a line is fitted between these two points. From this line, the centre of gravity can be found because it exists on this line and the distance of it from the centre points of the circle is known. Angle of attack is found using trigonometric relations for the line fitted. This process is repeated for each frame giving a time history of centre of gravity displacement and angle of attack. The distance between the two circles being a known quantity allows for the scale of the image $(0.69 \mathrm{~mm} /$ pixel $)$ to be calculated allowing the conversion from pixel displacement to displacement in metres. Figure 6 presents a composite image of a typical free-flight experiment with the image tracking algorithm detection points overlaid. Prior to testing, the spatial uniformity of the lens was measured and corrected for through the use of a uniform grid inserted into the tunnel.

To determine the aerodynamics coefficients, the displacement data is differentiated twice to give the accelerations and hence aerodynamic coefficients using Eq. 8 The intermediate velocity data required numerical smoothing using a Gaussian filter before being differentiated into accelerations.

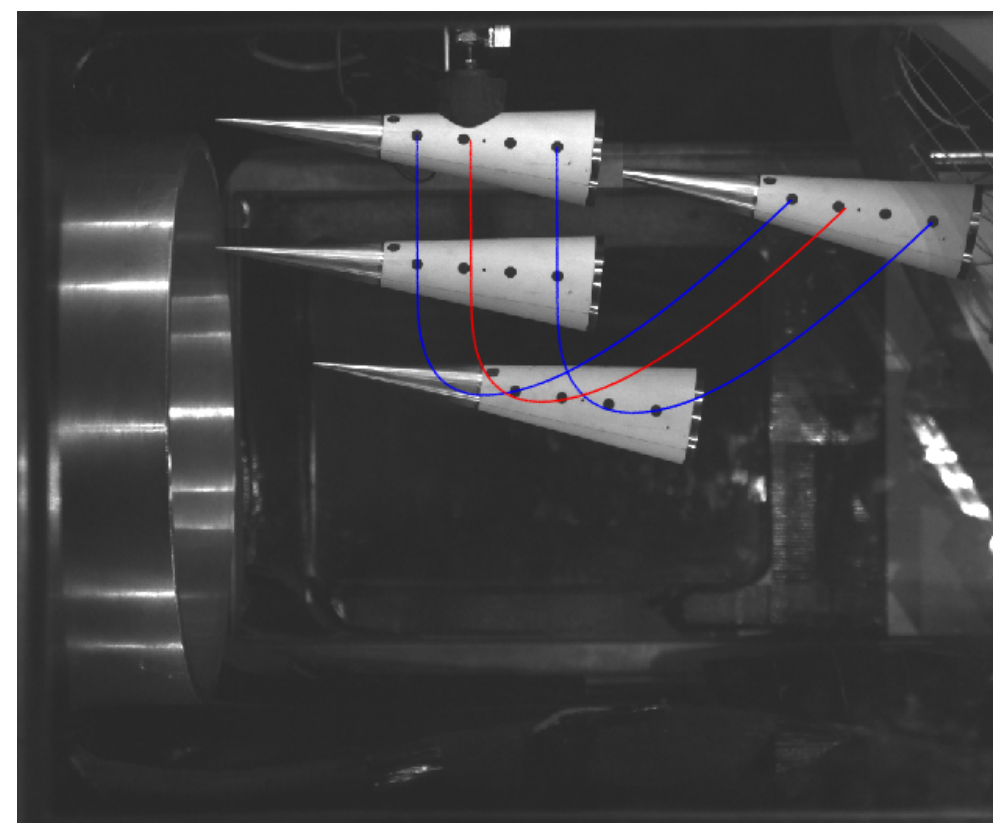

Fig. 6 Montage photo of image processing (Blue - detected circle centre points, Red - calculated centre of gravity).

The performance of the algorithm was analysed by calculating the position of centre of gravity for 500 frames where the model was held stationary. The standard deviation of centre of gravity position for this test was calculated to be $4 \mu \mathrm{m}$, which is very small considering the spatial resolution of the pixels $(0.69 \mathrm{~mm} / \mathrm{pixel})$ and the relative size of circles (radius of approximately 5 pixels) detected in relation to the overall size of the frame (1,024 x 1,024 pixels.). This analysis does not take into account errors which result from misalignment of the optical equipment but gives an approximation of the uncertainty associated with the numerical algorithm.

Yaw tracking has further complications when compared to longitudinal tracking because as the model falls, the distance between the yaw plane and the camera is not constant. To overcome this, the model was imaged with a smaller aperture of $\mathrm{f} 8$ to increase the depth of field. At this smaller aperture, it was necessary to decrease the frame rate to 1000 fps to expose the image correctly. Implementing yaw tracking was to enable determination of any out-of-plane motion that would impact the longitudinal aerodynamic coefficients 


\section{Angle of Attack Correction}

One consideration with free-flight experiments to be accounted for is the relative angle of attack of the model to the free-stream flow from the facility. The initial angle of attack for these experiments is determined from optical tracking. This however, could potentially result in a systematic error in angle of attack for all data points due to this unknown. For rigidly mounted, sting-based experiments, the experimenter will often use pressure transducers to measure the true zero degree angle of attack and use this subsequent position as a datum. This methodology becomes more difficult for free-flying models as on-board pressure sensors would be required.

To overcome this alignment issue, an alignment probe (four radial pressure transducers, north, east, south and west) was sting mounted to a two axis traverse and experiments were conducted to find the true zero angle of attack. This is at the point where the north and south pressure transducer read the same static pressure value. The optical cameras were used to calculate the angle of attack from their reference point and this offset was subsequently applied to all of the experimental data. The offset in angle of attack at Mach 6 and 7 was found to be $-0.59 \pm 0.05^{\circ}$ and $-0.57 \pm 0.05^{\circ}$ respectively for this test campaign. The camera set up remained fixed between free-flight tests and alignment test.

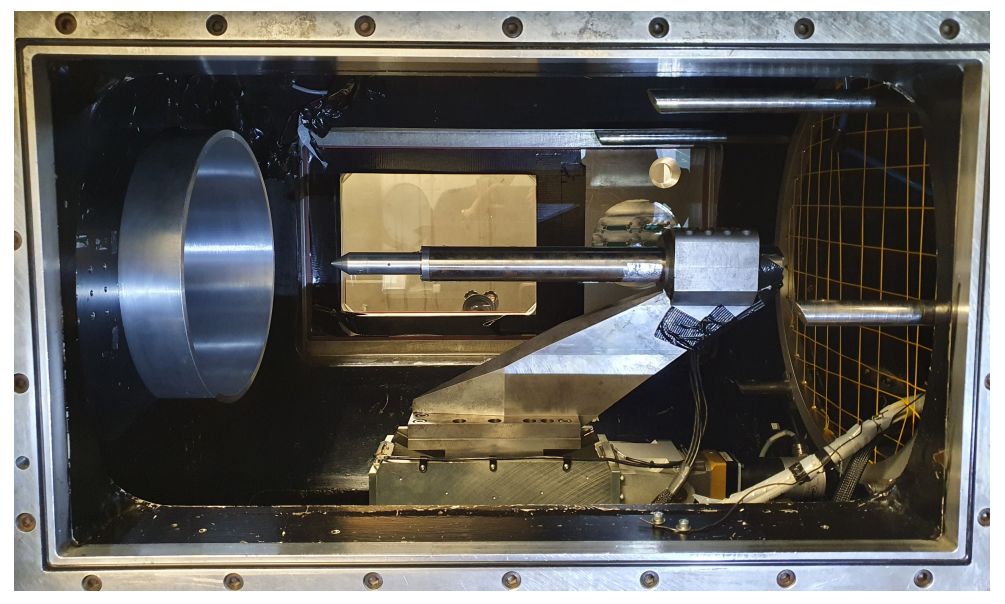

Fig. 7 Alignment probe installed in the Oxford High Density Tunnel 


\section{Results}

The results from the free-flight experiments are presented in this section. Lift, drag and pitching moment data are shown, as well as centre of pressure variation at the Mach 6 and 7 conditions. The experimental results are shown for both the IMU data and optical tracking and presented against viscous tangent cone predictions from the numerical panel method simulations. Uncertainties are shown as error bands around the data points as calculated using the Taylor Series Method determining the propagation of uncertainties in freestream parameters, model properties and the IMU as in Hyslop [8]. The maximum uncertainty in the coefficients for IMU data at Mach 6 and 7 is $2.52 \%$ and $2.55 \%$ respectively.

\section{A. Mach 6}

In total, 15 independent experiments were conducted at the Mach 6 test condition for the nominal cone geometry. Figure 8 presents the measured variation in lift coefficient with angle-of-attack, including a comparison with viscous tangent-cone calculations. The general trend of data is linear and agrees well with the numerical tangent-cone predictions. The linear trend gives a zero value for lift coefficient at $0^{\circ}$ angle of attack as expected for a axisymmetric body. The overall agreement between the image tracking and IMU data is also good for lift coefficient.

Figure 9 presents the drag coefficient against angle of attack for the Mach 6 condition. The non-viscous and viscous numerical predictions are plotted for comparison with the experimental data. The experimental data agrees well with the viscous numerical prediction and exhibits the expected parabolic shape. Figure 10 shows the lift to drag ratio against angle of attack which again the numerical predictions show good agreement with the experimental data.

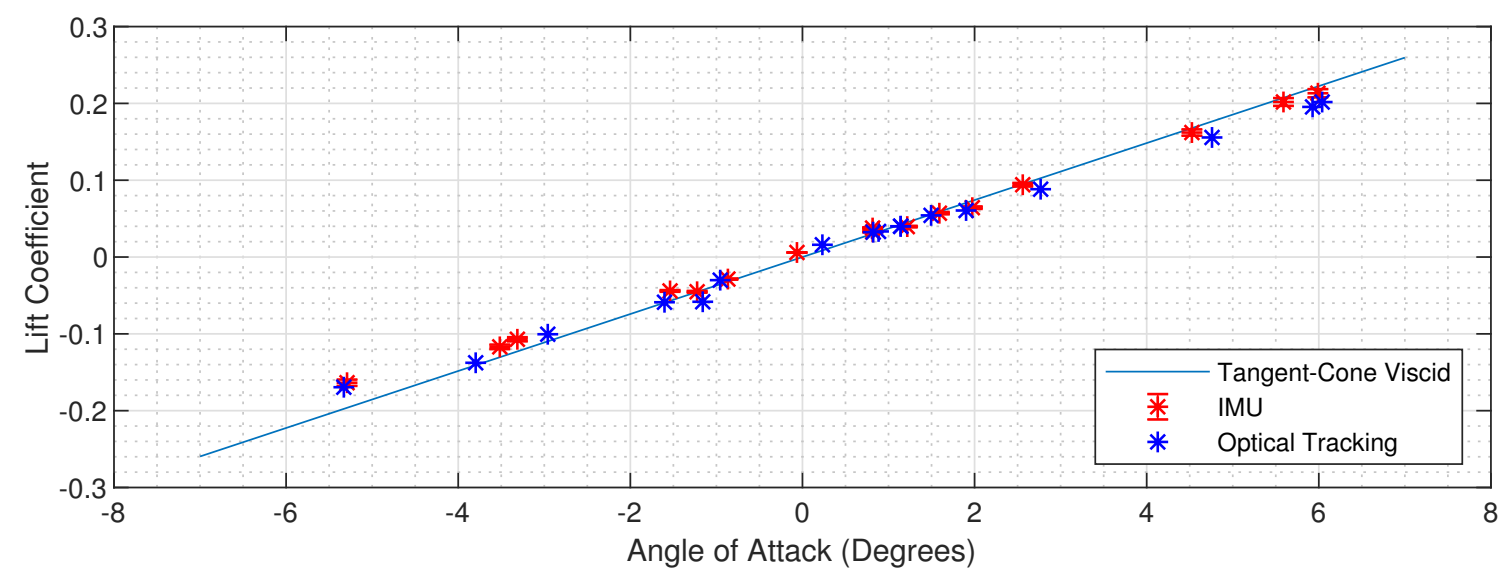

Fig. 8 Lift coefficient at Mach 6 condition. Individual tests plotted against tangent-cone numerical prediction

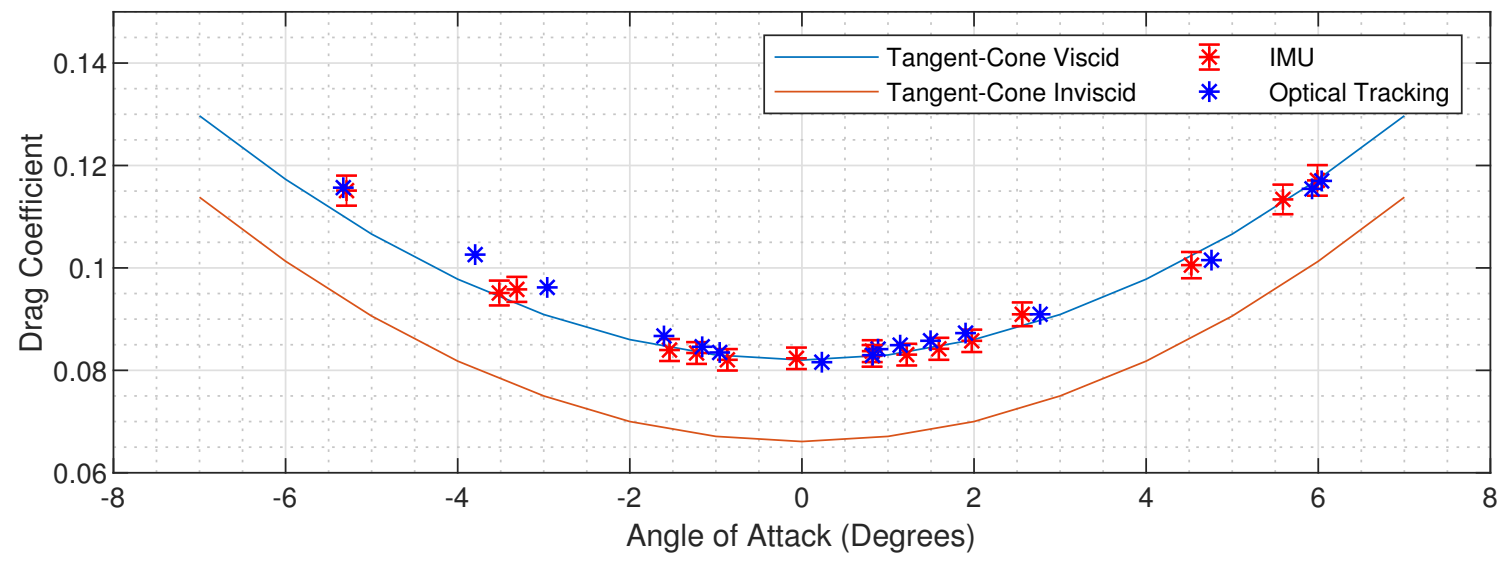

Fig. 9 Drag coefficient at Mach 6 condition. Individual tests plotted against tangent-cone numerical prediction 


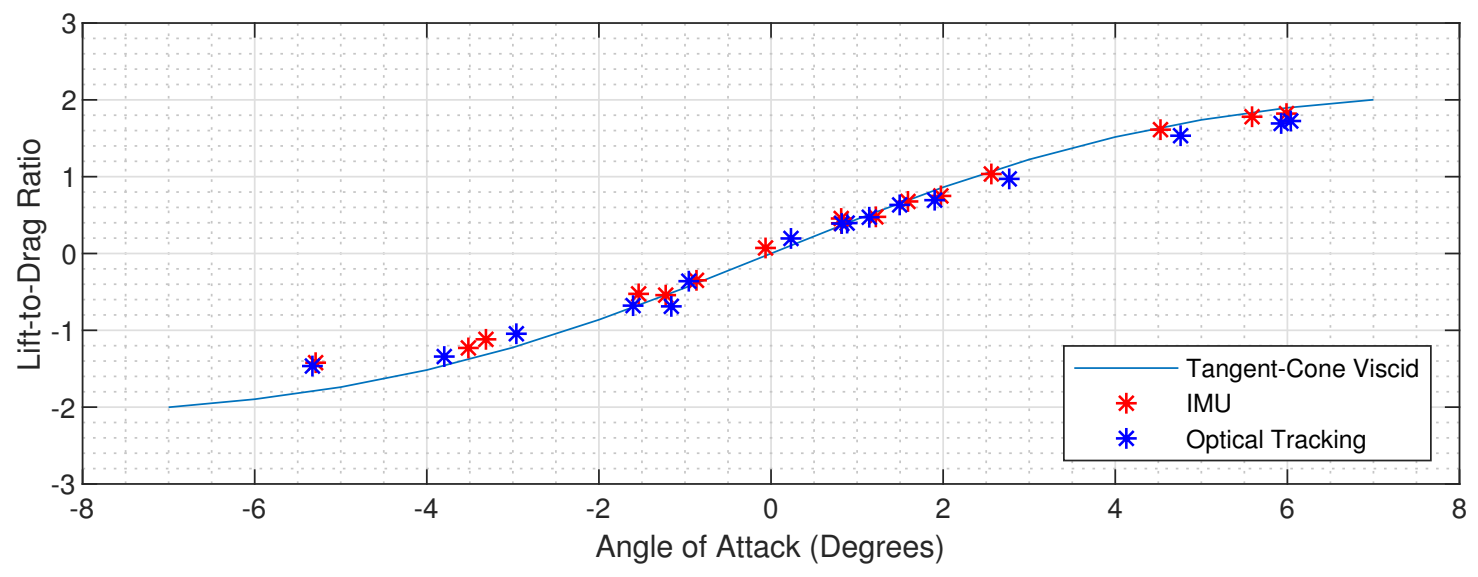

Fig. 10 Lift to drag ratio at Mach 6 condition. Individual tests plotted against tangent-cone numerical prediction

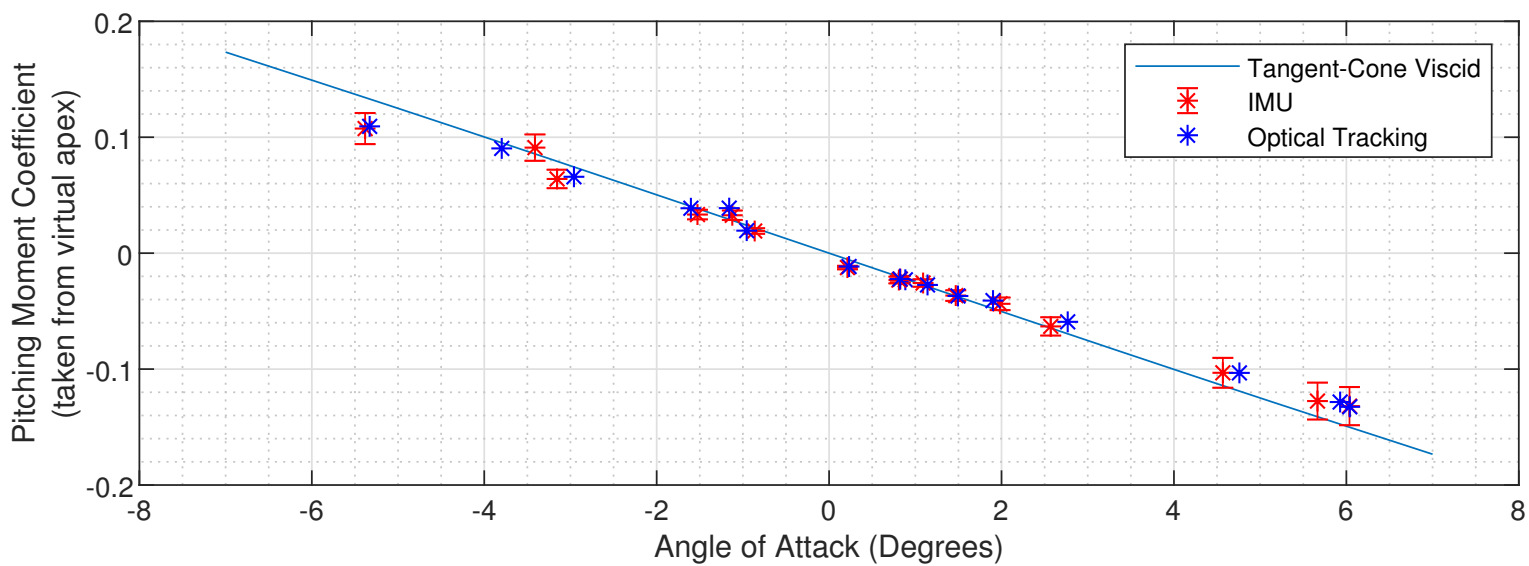

Fig. 11 Pitching moment coefficient at Mach 6 condition. Individual tests plotted against tangent-cone numerical prediction

Figure 11 presents the pitching moment coefficient variation with angle of attack referenced from the virtual apex of the cone. Referenced from this position, the plot shows a large degree static stability given by the negative gradient of the data. The numerical data begins to over predict at the extremities of the data but overall the agreement is good. The trim angle of attack is found to be $0^{\circ}$ angle of attack as to be expected. Centre of pressure, shown in Figure 12, is invariant with angle of attack. As all tests were conducted at angles of attack below the half angle of the cone, there was no separation on the leeward surface and so centre of pressure remains constant with angle of attack. All experimental data points were within $+/-4 \mathrm{~mm}$ ( $1.6 \%$ of the total length of the model) of the numerical tangent-cone predictions. 


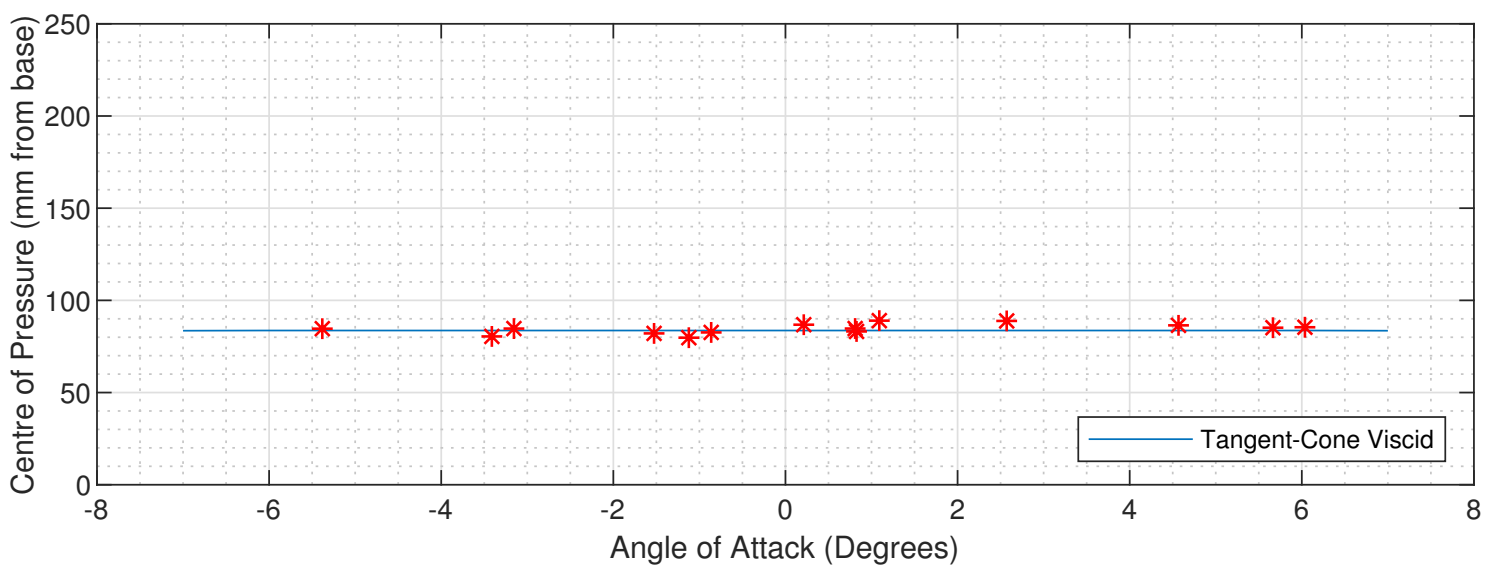

Fig. 12 Centre of Pressure at Mach 6 condition. Individual tests plotted against tangent-cone numerical prediction

\section{B. Mach 7}

In total, 12 independent tests were conducted at the Mach 7 test condition in Table 2. Results show very similar trends to the Mach 6 results with good agreement of the IMU data, optical tracking data and numerical panel code. Figures 13 and 14 present the lift and drag coefficient at the Mach 7 condition. There is a slight offset between the viscous numerical prediction and experimental data for drag coefficient. This is likely due to a large transient variation in total temperature during the test flow. The prediction of skin friction (hence viscous effects) is highly dependent on the temperature of the flow for numerical codes and so this is the most likely cause of this difference. Consequently, the numerical lift-to-drag ratio, presented in Figure 15, over predicts the experimental result at the extremities of angles of attack tested.

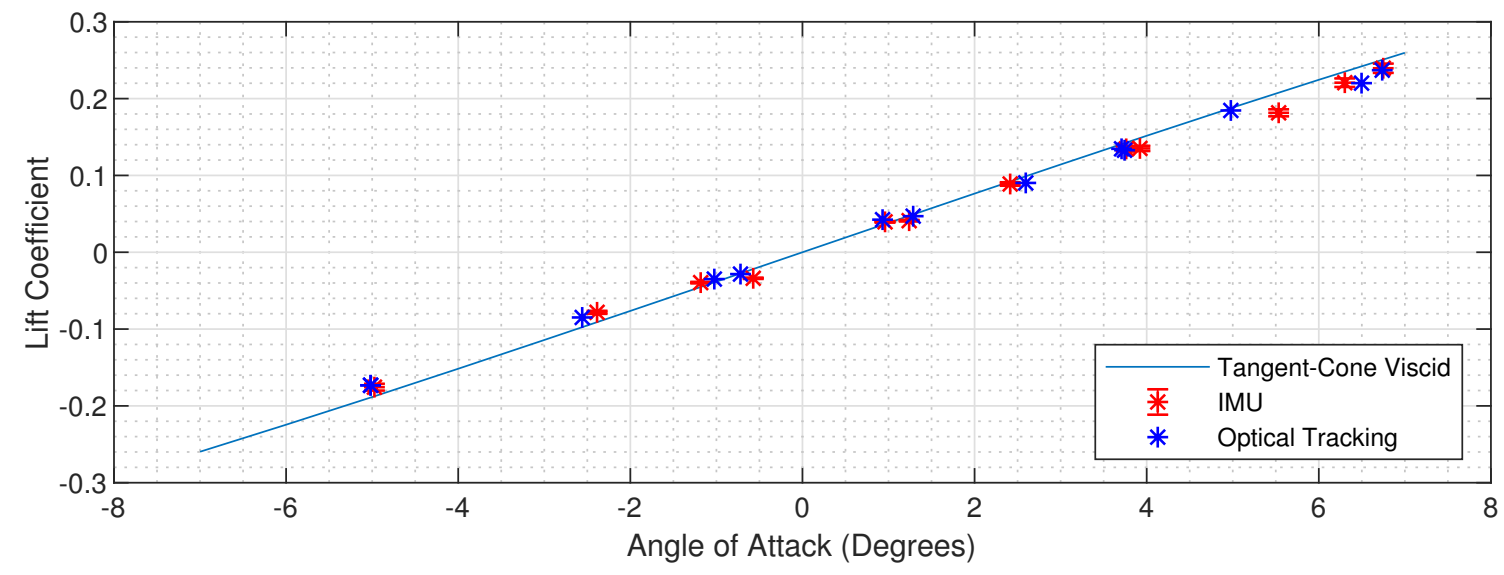

Fig. 13 Lift coefficient at Mach 7 condition. Individual tests plotted against tangent-cone numerical prediction 


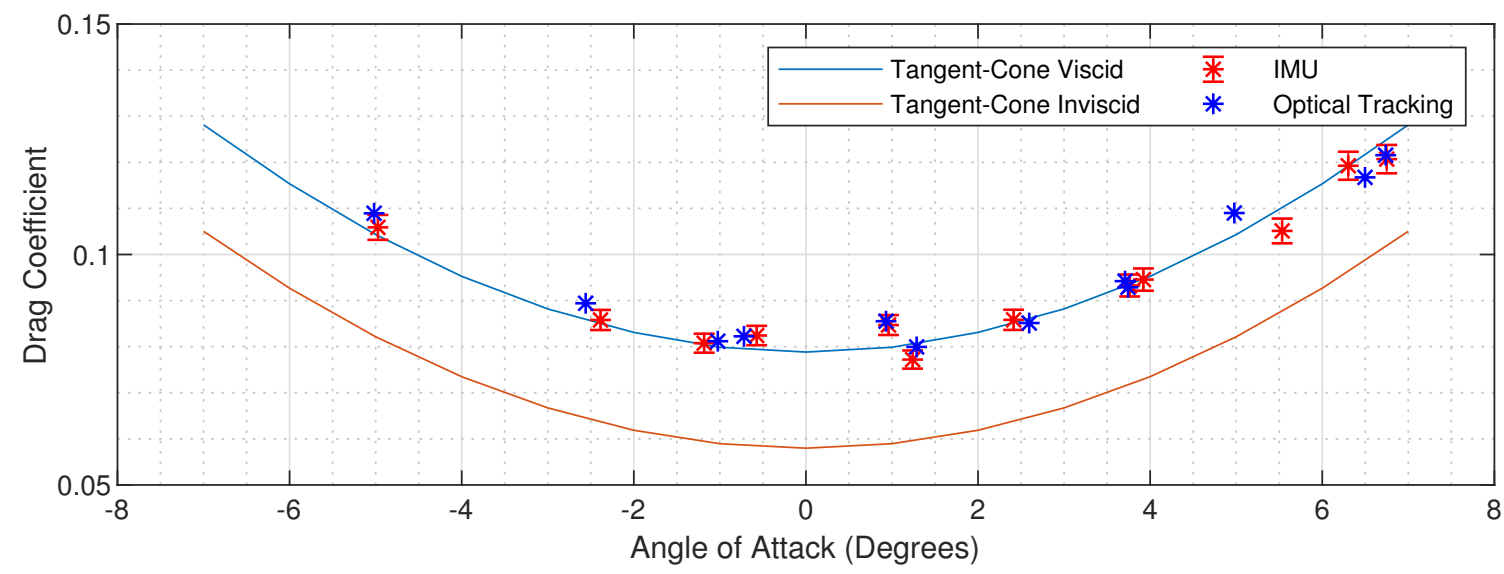

Fig. 14 Drag coefficient at Mach 7 condition. Individual tests plotted against tangent-cone numerical prediction

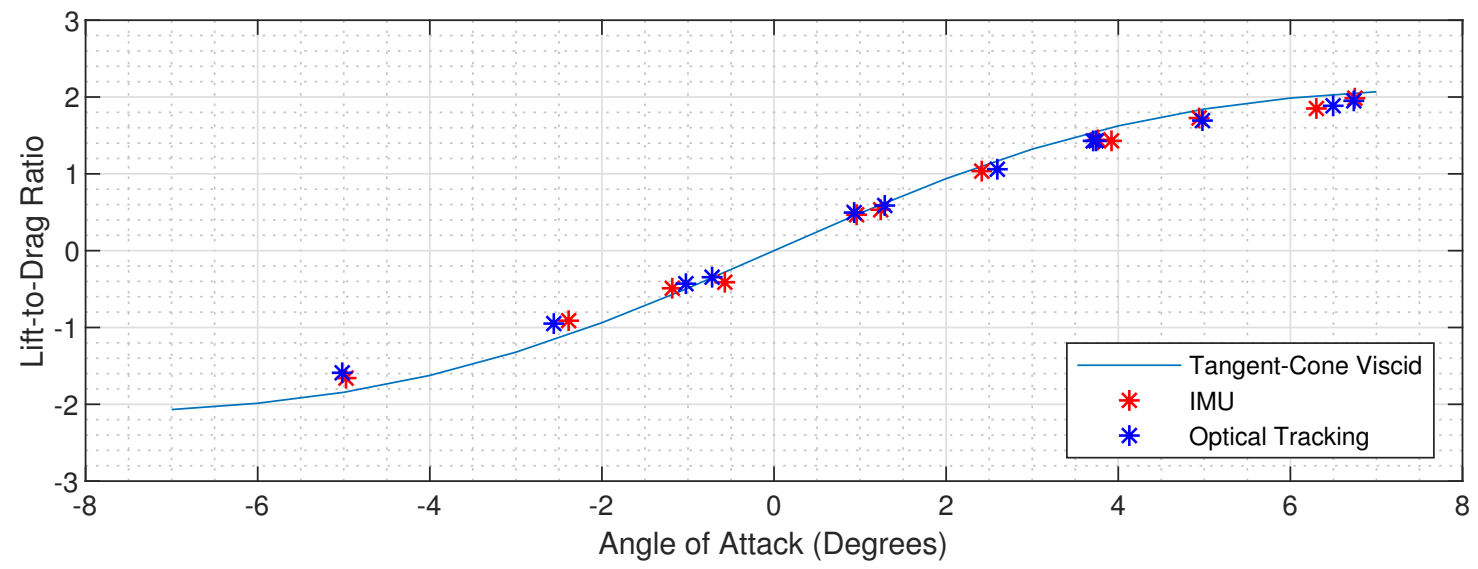

Fig. 15 Lift to drag ratio at Mach 7 condition. Individual tests plotted against tangent-cone numerical prediction

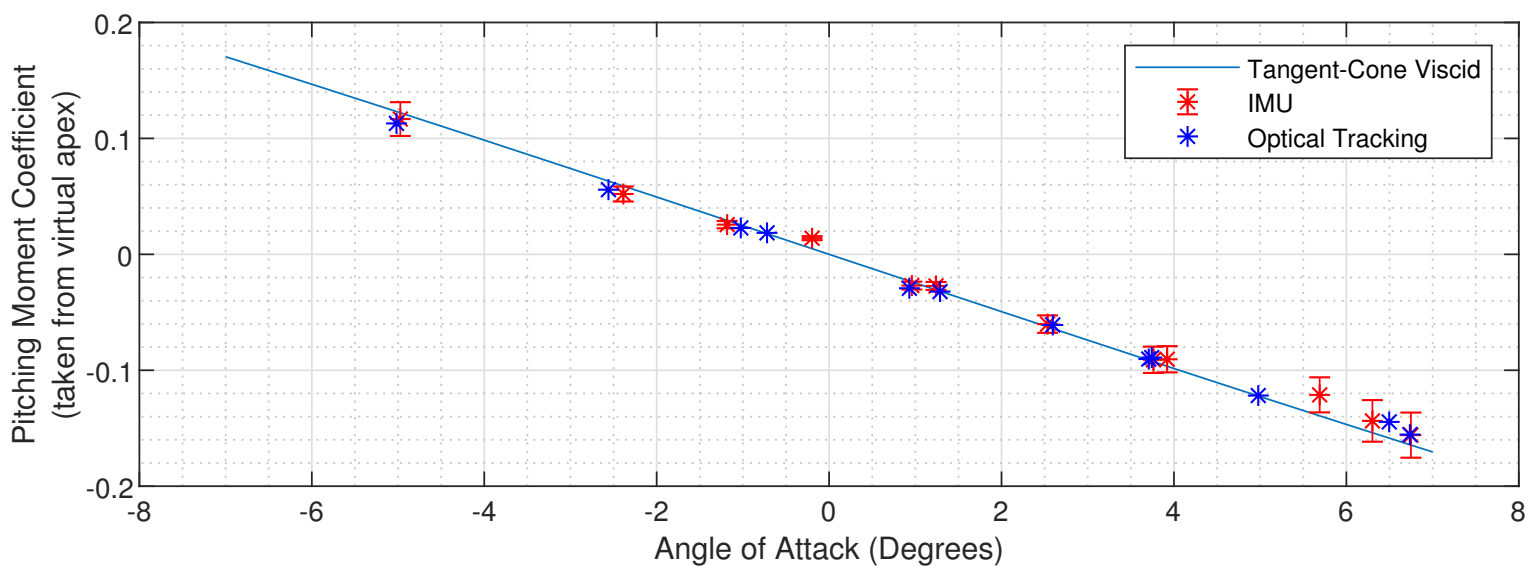

Fig. 16 Pitching moment coefficient at Mach 7 condition. Individual tests plotted against tangent-cone numerical prediction

The pitching moment coefficient presented in Figure 16 exhibits the static stability when referenced from the nose and the agreement between experimental and numerical data is good. Location of centre of pressure is also invariant with angle of attack and takes on a similar value to the Mach 6 data suggesting a degree of Mach number independence. 


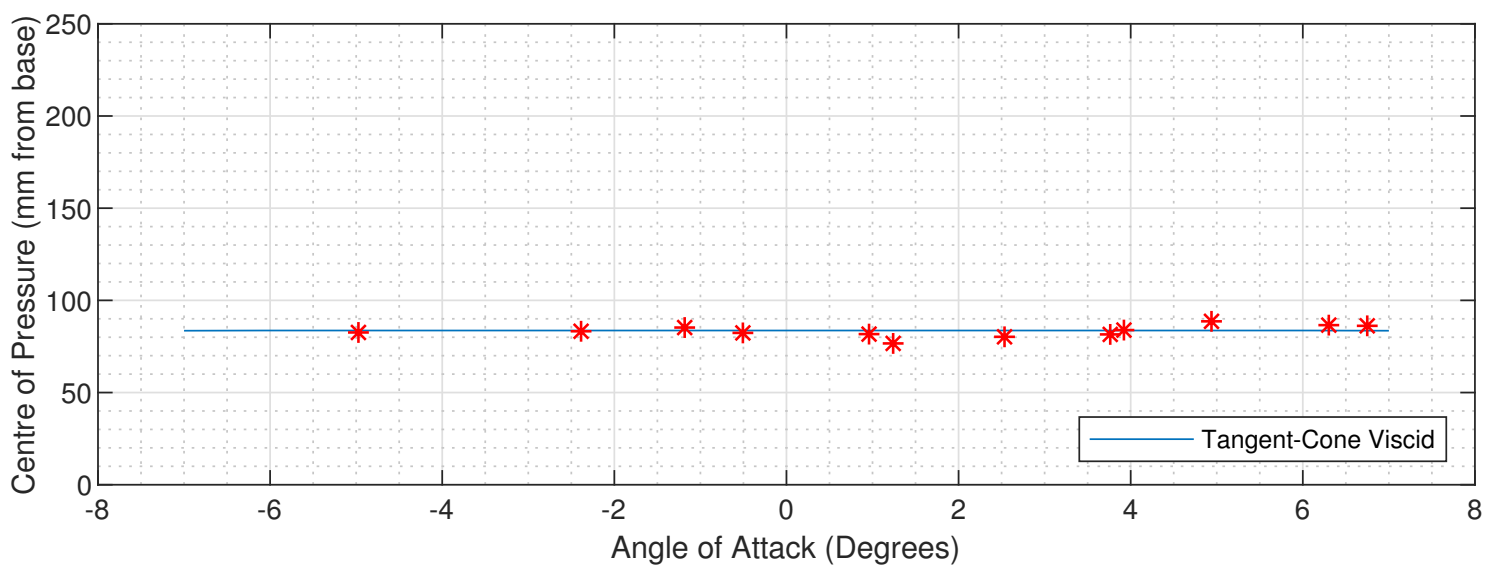

Fig. 17 Centre of pressure at Mach 7 condition. Individual tests plotted against tangent-cone numerical prediction

\section{Flow Visualisation}

Schlieren imagery was used to visualise the flow around the cone model for 2 independent shots. To achieve this, the image tracking system was removed due to the limitation of optical access to the test section. The purpose of using schlieren imagery was two-fold; firstly it confirmed that the model was in the core flow of the nozzle for the duration of the tests and secondly, it confirmed that no unusual flow phenomena were present that would affect the force coefficients. Unfortunately, the whole cone model cannot be seen in one frame due to a limitation of mirror size and consequent elliptical field of view. Figure 18 shows a series of images from free-flight test. The first image is prior to flow arrival with the model in free-fall and the subsequent three images are with flow on. As expected, the images show a bow shock from the nose tip and expansion fans over the model base. The images also confirm that the model remains in the core flow for the duration of the test.

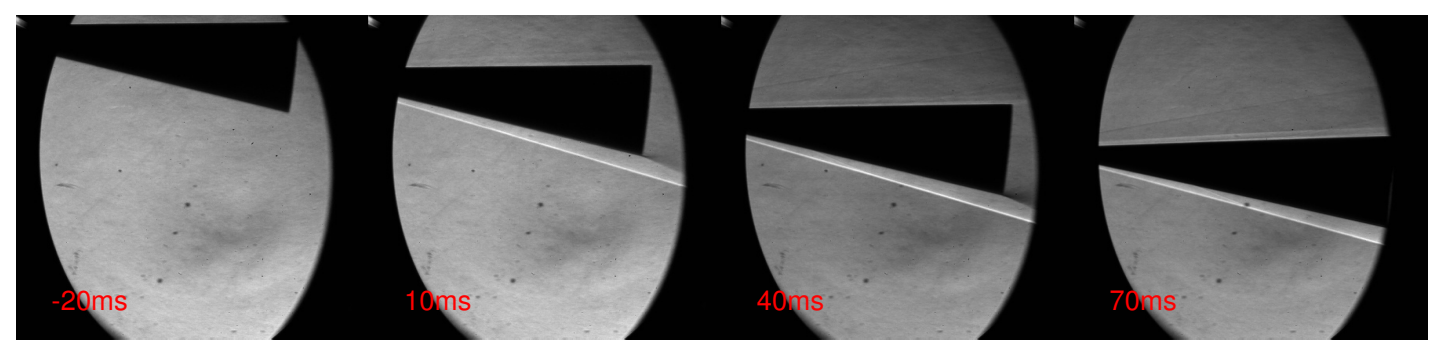

Fig. 18 Schlieren imagery of cone model in free-flight at Mach 7. Time at $0 \mathrm{~ms}$ refers to flow arrival.

\section{Summary and Conclusions}

The experimental measurement of static lift, drag and pitching moment coefficients have been presented for a 7 degree half angle cone at a Mach 6 and 7 test condition representative of an altitude of $35 \mathrm{~km}$. The use of a model with minimal static margin has been shown to be effective at determining static aerodynamic coefficients with very low uncertainties that have been validated against numerical simulations. Lift and drag coefficient exhibited the expected shapes with respect to angle of attack with drag coefficient being notably influenced by viscous effects. Furthermore, the two independent methods of measuring accelerations, image tracking and an on-board IMU, show very good agreement in the experimental data, adding redundancy to the technique. Centre of pressure was also shown to be invariant with angle of attack and in good agreement with the panel code. Overall, the quasi-static free-flight technique has been 
validated for a simple 7 degree half-angle cone which gives a high degree of confidence for obtaining static aerodynamic coefficients for more complex hypersonic vehicles.

\section{Acknowledgments}

The authors would like to acknowledge the exhaustive work of Mailys Buquet for operating the HDT during testing. We would also like to thank Greg King for his precise work painting the cone model. We would also like to thank Prof. Andrew Neely and Mr Liam McQuellin from UNSW Canberra for supplying the free-flight DAQs. Finally, we would like to thank Trevor Birch of DSTL for his advice and support during the project.

\section{References}

[1] Wuilbercq, R., Pescetelli, F., Minisci, E., and Brown, R. E., "Influence of Boundary Layer Transition on the Trajectory Optimisation of a Reusable Launch Vehicle," 19th AIAA International Space Planes and Hypersonic Systems and Technologies Conference, 2014, p. 2362. https://doi.org/https://doi.org/10.2514/6.2014-2362

[2] Stetson, K. F., "Hypersonic boundary-layer transition,” Advances in Hypersonics, Springer, 1992, pp. 324-417.

[3] Juhany, K. A., and Darji, A., "Force Measurement in a Ludwieg Tube Tunnel," Journal of Spacecraft and Rockets, Vol. 44, No. 1, 2007, pp. 88-93. https://doi.org/10.2514/1.21014

[4] Mee, D. J., Daniel, W. J. T., and Simmons, J. M., “Three-Component Force Balance for Flows of Millisecond Duration,” AIAA Journal, Vol. 34, No. 3, 2008, pp. 590-595. https://doi.org/10.2514/3.13108

[5] Pick, G. S., "Sting Effects in Hypersonic Base Pressure Measurements,” Tech. rep., TR AL-85, Dec. 1971, Naval Ship Research and Development Center , Bethesda, Md., 1971.

[6] Kennell, C., Neely, A. J., O’Byrne, S. B., and Buttsworth, D., "Measurement of Vehicle Stability Coefficients in Hypersonic Wind Tunnels," 20th AIAA International Space Planes and Hypersonic Systems and Technologies Conference, 2015 , p. 3690. https://doi.org/https://doi.org/10.2514/6.2015-3690

[7] Kennell, C., Neely, A. J., Buttsworth, D. R., Choudhury, R., and Tahtali, M., "Free Flight Testing in Hypersonic Flows: HEXAFLYINT EFTV,” 54th AIAA Aerospace Sciences Meeting, 2016, p. 1152. https://doi.org/https://doi.org/10.2514/6.2016-1152

[8] Hyslop, A., Mcgilvray, M., Hermann, T., Doherty, L. J., Neely, A. J., Mcquellin, L., Barth, J., and Mullen, G., “Aerodynamic Testing Skylon Space Plane of the," 2019, pp. 1-11.

[9] Wolowicz, C. H., Bowman, J. S., and Gilbert, W. P., "Similitude Requirements and Scaling Relationships as applied to Model Testing," Tech. Rep. 1435, NASA, 1979.

[10] Hunter, D., “UK Hypersonic Glide Vehicle Concept and Performance Assessment,”, No. November, 2019.

[11] McGilvray, M., Doherty, L. J., Neely, A. J., Pearce, R., and Ireland, P., “The Oxford High Density Tunnel," 20th AIAA International Space Planes and Hypersonic Systems and Technologies Conference, 2015, p. 3548. https://doi.org/https: //doi.org/10.2514/6.2015-3548

[12] Wylie, S., Doherty, L., and McGilvray, M., "Commissioning of the Oxford High Density Tunnel (HDT) for Boundary Layer Instability Measurements at Mach 7,” 2018 Fluid Dynamics Conference, 2018, p. 3074. https://doi.org/https://doi.org/10.2514/ 6.2018-3074

[13] Acton, J. M., “Hypersonic boost-glide weapons," Science and Global Security, Vol. 23, No. 3, 2015, pp. $191-219$. https://doi.org/10.1080/08929882.2015.1087242

[14] McQuellin, L. P., Kennell, C. M., Neely, A. J., Sytsma, M. J., Silvester, T., Choudhury, R., and Buttsworth, D. R., "Investigating endo-atmospheric separation of a hypersonic flyer-sustainer using wind tunnel based free-flight," 23rd AIAA International Space Planes and Hypersonic Systems and Technologies Conference, 2020, 2020, pp. 1-24. https://doi.org/10.2514/6.2020-2451

[15] Donaldson, N. L., and Ireland, P., "A Panel Method Aerodynamic Preprocessor for Planetary Entry Trajectory Simulations," 21st AIAA International Space Planes and Hypersonics Technologies Conference, 2017, p. 2379.

[16] Oceanic, U. S. N., Administration, A., and Force, U. S. A., US standard atmosphere, 1976, Vol. 76, National Oceanic and Atmospheric Administration, 1976. 
[17] Cruz, C. I., and Sova, G. J., "Improved tangent-cone method for the Aerodynamic Preliminary Analysis System (APAS) version of the hypersonic arbitrary-body program," 1990.

[18] Anderson Jr, J. D., Hypersonic and high-temperature gas dynamics, American Institute of Aeronautics and Astronautics, 2006.

[19] Wuilbercq, R., and Brown, R. E., "Rapid Aero-Thermodynamic Analysis for Hypersonic Air Vehicles," Proceedings of the 8th European Symposium on Aerothermodynamics for Space Vehicles, 2015.

[20] Meador, W. E., and Smart, M. K., "Reference enthalpy method developed from solutions of the boundary-layer equations," AIAA journal, Vol. 43, No. 1, 2005, pp. 135-139.

[21] Eckert, E. R. G., "Engineering relations for heat transfer and friction in high-velocity laminar and turbulent boundary-layer flow over surfaces with constant pressure and temperature," Transactions of the ASME, Vol. 78, No. 6, 1956, pp. $1273-1283$.

[22] Canny, J., "A Computational Approach to Edge Detection," IEEE Transactions on Pattern Analysis and machine Intelligence, Vol. PAMI-8, No. 6, 1986, pp. 679-698.

[23] Hough, P. V. C., "Method and Means for Recognizing Complex Patterns,”, dec 1962.

[24] von Gioi, R. G., and Randall, G., "A Sub-Pixel Edge Detector: an Implementation of the Canny/Devernay Algorithm.” IPOL Journal, Vol. 7, 2017, pp. 347-372.

[25] Laurence, S. J., and Hornung, H. G., "Image-Based Force and Moment Measurement in Hypersonic Facilities," Experiments in Fluids, Vol. 46, No. 2, 2009, pp. 343-353. 